\title{
The application of micropulse transscleral contact- compression diode-laser cyclocoagulation for emergency reduction of intraocular pressure in patients with glaucoma
}

\begin{abstract}
We analyzed the efficacy, safety and speed reduction of IOP which cannot be corrected by other methods by a single threshold (MTCCLCC) procedure in patients with different forms of glaucoma. After one session of MTCCDLC IOP decreased on average by $11.39 \mathrm{mmHg}$.

The relative decrease in IOP was $33.0 \%$ for the group of patients with POAG group and $27.6 \%$ for neovascular glaucoma. The overall success rate for all patients was $52 \%$. The achieved efficacy and the absence of serious complications indicate that threshold MTCCLCC can be widely used in clinical practice as a means of treatment for a quick reduction in IOP.
\end{abstract}

Keywords: glaucoma, micropulse transscleral cyclocoagulation, laser, IOP
Volume 8 Issue 2 - 2018

\section{Kliuiev GO, Privalov AP, Luhova OM \\ Health Technology Ltd, Ukraine}

Correspondence: Kliuiev GO, Health Technology Ltd, Ukraine, Email gkluev@kluev.com.ua

Received: March 27, 2018 | Published: April 6, 2018

\section{Introduction}

Glaucoma was and remains one of the most relevant problems of ophthalmology. The percentage of blindness and low vision as a result of glaucoma remains stable and does not tend to decrease. ${ }^{1}$ Despite modern advances in drug therapy for glaucoma, in many cases, only laser and surgical treatment makes it possible to achieve a stable normalization of intraocular pressure and stabilize visual functions, and for so-called refractory glaucoma, laser surgery is generally a method of choice. In recent years, the arsenal of methods for the treatment of glaucoma has expanded due to the use of modern semiconductor lasers for transscleral cyclocoagulation. ${ }^{2,3}$

In the pathogenesis of glaucoma involved numerous factors mechanical, vascular, metabolic. However, exactly the increase of IOP has a direct damaging effect on the structure of the optic nerve disc and leads, ultimately, to irreversible blindness. In severe cases, when the intraocular pressure cannot be reduced with maximum medication, and surgical treatment is associated with an increased risk due to possible complications, the use of laser transscleral cyclocoagulation is the method of choice. ${ }^{4}$ This method allows to reduce IOP quickly and effectively. However, the use of standard methods of laser exposure is fraught with significant complications, such as the development of hypotension, phthisis, hyphema, vitreous hemorrhage, uveitis, inflammation and other serious complications. ${ }^{5-7}$

Since 2006, we have begun to apply for these purposes micropulse transcleral contact-compression diode laser cyclocoagulation (MTCCDLC) with using a semiconductor laser at a wavelength of $810 \mathrm{~nm}$. The MTCCDLC method allowed to increase safety and significantly reduce the level of complications. ${ }^{8}$ The increase in the number of patients requiring urgent reduction of ophthalmotonus and having a high risk of surgical intervention led us to study the possibility of using laser cyclocoagulation as an emergency procedure to reduce IOP. The aim of the research was to study the possibility of quickly, effectively and safely reducing IOP not corrected by other methods, a single procedure of MTCCDLC in patients with different forms of glaucoma.

\section{Materials and methods of research}

The research was conducted in 109 patients ( 115 eyes), the average age of which is $65.33 \pm 13.75 .52$ of them were women and 57 of them were men. All patients received the maximum medication. MTCCDLC was used in cases when drug therapy did not lead to normalization of IOP or there were contraindications to surgical treatment. There was used the coagulator developed by us for transcleral cyclocoagulation problems with a semiconductor laser with a wavelength of $810 \mathrm{~nm}$. The coagulator is adapted with a quartz-polymer fiber with a diameter of $600 \mu \mathrm{m}$. The design of the transscleral probe provided a smooth change in the force of $0.1-0.5 \mathrm{~N}$ with the adjustment screw of the spring device.

The axis of the transscleral probe was oriented perpendicular to the scleral surface and deviated from the optic axis of the eye. Initial increase in fiber pressure was established by $0.1 \mathrm{~N}$ and increased for elderly patients. Cyclocoagulation was performed under combined epibulbar anesthesia with $0.5 \%$ alkane (3 fold installation) and parabulbar anesthesia with marcaine $(5 \mathrm{mg} / \mathrm{ml})-3 \mathrm{ml}$.

\section{The results obtained and their discussion}

Using the Mann-Whitney test, IOP was compared before surgery with IOP of the remaining time intervals.

$\mathrm{M} \pm \mathrm{m}$-average value with standard error, $\mathrm{N}-$ number of eyes, Min/ Max-minimum/maximum value of IOP. 
The Mann-Whitney test showed that statistically significant (significance level $\mathrm{p}<0.05$ ) is IOP a day after the operation and IOP at discharge, i.e. there is a difference between IOP in patients diagnosed with NVG and POAG. That means, that in the group of patients with NVG and POAG, a significant difference is present between the initial
IOP, the pressure measured one day after the operation and IOP at the end of the time. If to pay attention to the indicators of mean values, then after one session of MTCCDLC, IOP decreased on average 11.39 $\mathrm{mmHg}$ (Figure 1).

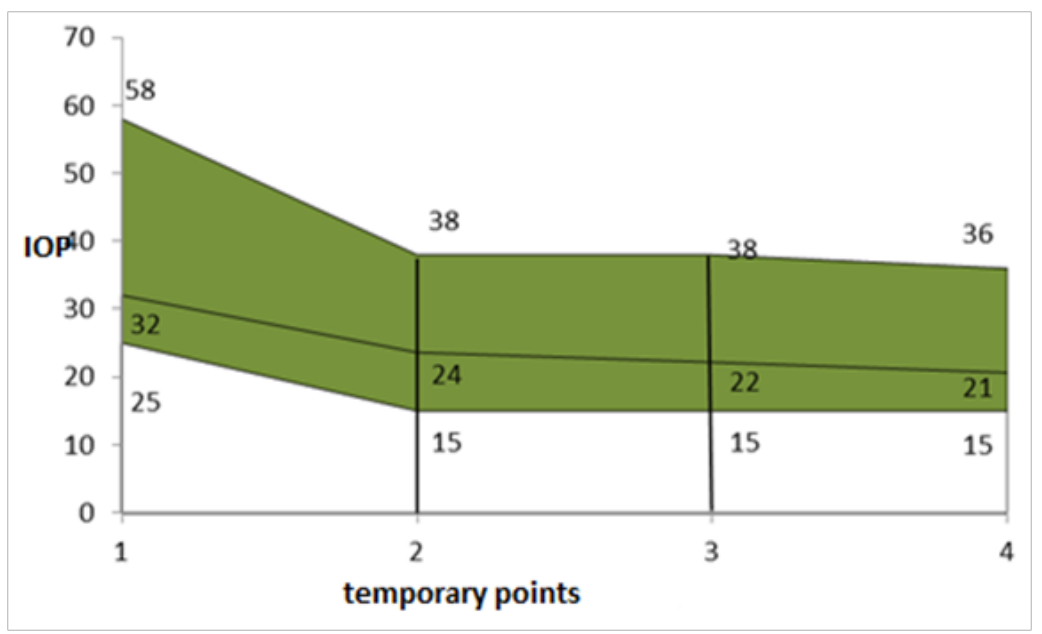

Figure I *I-IOP before operation, 2-IOP after one day of operation, 3 - IOP after some days of operation, 4 - IOP discharge.

Table I Change in IOP in patients with diagnoses POAG and NVG after MTCCDLC

\begin{tabular}{lllllll}
\hline & $\begin{array}{l}\text { Average value } \\
\text { M } \pm m\end{array}$ & N & Min & Max & Standard deviation & Significance level $\mathbf{p}^{*}$ \\
\hline IOP before & $32,03 \pm 0,58$ & 115 & 25 & 58 & 6,61 & \\
IOP after one day & $23,62 \pm 0,53$ & 108 & 15 & 38 & 5,74 & 0,013368 \\
IOP intermediate & $22,18 \pm 0,58$ & 91 & 15 & 38 & 5,83 & 0,072278 \\
IOP discharge & $20,64 \pm 0,36$ & 115 & 15 & 36 & 4,06 & 0,016623 \\
Energy & $48,75 \pm 2,93$ & 111 & 20,00 & 116,00 & 23,37 & \\
\hline
\end{tabular}

The relative decrease in IOP was $33.0 \%$ for the POAG group and $27.6 \%$ for the neovascular glaucoma. The overall success rate for all patients was $52 \%$. At an average energy in the pulse of $1.3 \mathrm{~J}$, the average summary radiation energy per session was $39.6 \mathrm{~J}$, which is lower than the energies used by standard methods. The manifestation of complications that are caused by explosive effects arising from the use of elevated capacities in the implementation of standard cyclocoagulation techniques have been virtually eliminated, since the power and average energy in the pulse for MTCCDLC are much lower than necessary for the manifestation of these effects.

\section{Conclusion}

Micropulse transcleral contact-compression diode laser cyclocoagulation (MTCCDLC) with the achieved efficacy and the absence of severe complications can be widely used in clinical practice as an emergency aid for the rapid reduction of IOP in patients with different forms of glaucoma with high IOP, not corrected by medical therapy.

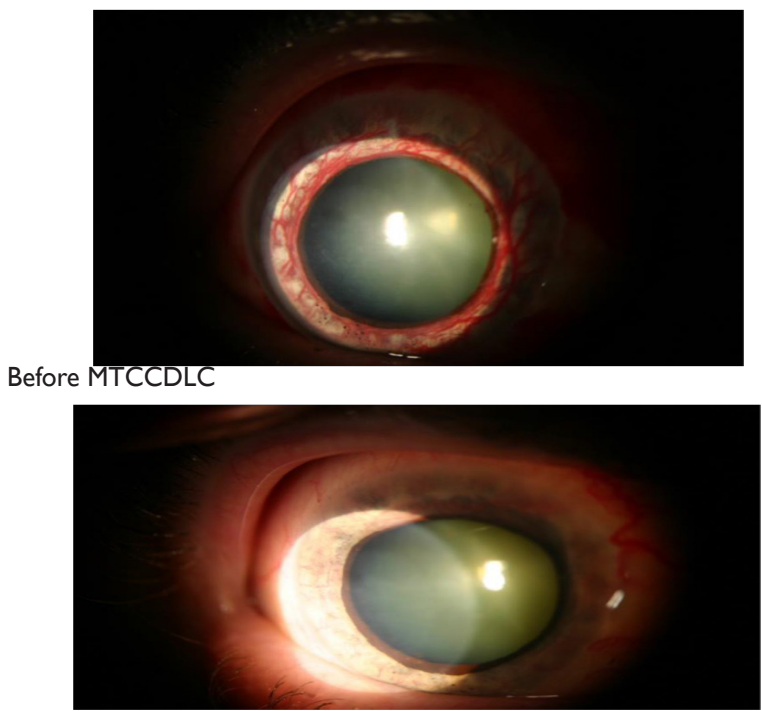

After MTCCDLC 


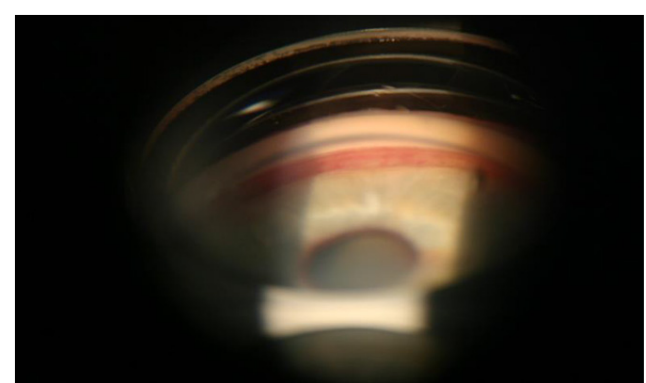

Before MTCCDLC

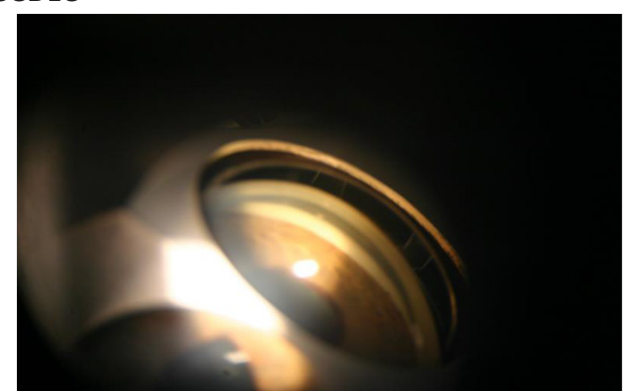

After MTCCDLC

\section{Acknowledgments}

None.

\section{Conflict of interest}

The author declares there is no conflict of interest.

\section{References}

1. Quigley HA, Broman AT. The number of people with glaucoma worldwide in 2010 and 2020. Br J Ophthalmol. 2006;90(3):262-267.

2. Bloom PA. Ciliary body laser treatment for glaucoma: a contemporary view. Glaucoma world. 2000;5(20):10-17.

3. Schlote T, Derse M, Rassman K. Efficacy and safety of contact transscleral diod laser cyclophotocoagulation for advanced glaucoma. $J$ glaucoma. 2001;10(4):294-301.

4. Gaasterland DE, Pollack IP, Gaasterland DE. Initial experience with a new method of laser transscleral cyclophotocoagulation for ciliary ablation in severe glaucoma. Trans Am Ophthalmol Soc. 1992;90:225-46.

5. Volkov VV. Transscleral diodmicrolaser cyclophobic coagulation in the treatment of complicated forms of glaucoma. Volkov VV, Kachanov AB, 6th congress of ophthalmologists in Russia: abstracts. M. 1994. 215 p.

6. Kachanov AB. Diode-laser transscleral contact cyclocoagulation in the treatment of various forms of glaucoma and ophthalmic hypertension: author's abstract. diss. for the competition uch. degree of Cand. Honey. Science. 995:21.

7. Egbert PR, Fiadoyor S, Budenz DL. Diod transscleral cyclophotocoagulation as a primary surgical treatment for primary openangle glaucoma. Arch Ophthalmol. 2001;119(3):345-350.

8. Klyuyev GO. Compression transscleral probes for laser cyclocoagulation. Ophthalmol. Journal. 2007;6:62-65. 